Self-Explanation and Digital Games:

Adaptively Increasing Abstraction

\author{
Douglas B. Clark ${ }^{1}$ \\ Satyugjit S. Virk ${ }^{1}$ \\ Jacqueline Barnes $^{2}$ \\ Deanne M. Adams ${ }^{3}$
}

\author{
Vanderbilt University ${ }^{1}$ \\ Northeastern University ${ }^{2}$ \\ Microsoft Corporation ${ }^{3}$
}

Author Note

Douglas B. Clark, Department of Teaching and Learning, Vanderbilt University.

Satyugjit S. Virk,Department of Teaching and Learning, Vanderbilt University.

Jacqueline Barnes, College of Education, Northeastern University

Deanne M. Adams, Games Testing and Research, Microsoft Corporation

The learning environment, study, and data reported here were supported by the Institute of Education Sciences, U.S. Department of Education, through Grant R305A110782 to Vanderbilt University. The analyses, writing, and reporting of the data and study were supported by the National Science Foundation through grant 1119290 to Vanderbilt University. The opinions expressed are those of the authors and do not represent views of the Institute, the U.S. Department of Education, or the National Science Foundation.

Correspondence concerning this article should be addressed to Douglas Clark, Box 230, 230 Appleton Place, Nashville, TN 37203-5721, USA

Contact: doug.clark@vanderbilt.edu 


\title{
Self-Explanation and Digital Games: Adaptively Increasing Abstraction
}

\begin{abstract}
Research suggests thatself-explanation functionality can effectively support learningin the context of digital games. Research also highlights challenges, however,in balancing and integrating the demands and abstraction of self-explanation functionality with the demands and structure of the game. These challenges are particularly true for games that are, themselves, cognitively more complex. The current study presents an approach that adapts the abstraction of self-explanation prompts based on a player's performance. The results demonstrate that students in this condition (a) scored significantly higher on the post-test than students whose selfexplanation prompts were not adaptively adjusted and were always abstract and (b) scored higher, but not significantly so, than students who did not receive the self-explanation functionality. Analyses of gameplay metrics suggest that trade-offs in terms of progress through the game may explain some aspects of these post-test comparisons. Analyses also demonstrate that both self-explanation conditions significantly outperformed the navigation-only comparison conditionon agameplay metric that suggests deeper model-based thinking.
\end{abstract}

Key Words. Self-Explanation, Digital Games, Science Education

\section{Introduction}

Prompting students to engage in self-explanation can enhance learning by encouraging students to engage in meta-cognitive activities to monitor what they do and do not understand (Chi, Bassok, Lewis, Reimann, \& Glaser 1989; Roy \& Chi, 2005; Chi \& VanLehn, 1991). 
Previous research demonstrated, however, that incorporatingquestions to prompt selfexplanationin a digital game can disrupt cognitive and gameplay flow in a manner that results in fewer game levels completed and no significant increases in performance (Authors, 2014). O’Neil et al. (2014) similarly concluded that, while self-explanation prompts cansupport generative processing, self-explanation prompts can also result in(a) extraneous processing by slowing down the player and distracting the player from learning or (b) minimal processing due to the playerignoring the prompts and trying to return to gameplay as quickly as possible. These findings contrasted with other previous findings stating that self-explanation and explanatory prompts can enhance learning in digital games (Moreno \& Mayer, 2005, Mayer \& Johnson, 2010, Johnson \& Mayer, 2010; Hsu, Tsai, \& Wang, 2012).

Together, previous research suggested thatself-explanation functionality can be effective in games for learning, but this previous research also illuminated the challenges in balancing and integrating the demands and abstraction of self-explanation functionality with the demands of the game, particularly for games that are themselves cognitively more complex. Accordingly, the current study presents an approach that adapts the abstraction of the self-explanation prompts based on a player's performance. More specifically, the purpose of this study is to explore whether adapting the abstraction of self-explanation prompts based on students' performancemight minimize cognitive and/or gameplay disruptionand thus enhance learning and engagement. This study also explores how such prompts might differentially affect game play behaviorand how variations in game play behavior might relate to variations in outcomes on conceptual assessments.

In support of these goals, the following structure is employed in presenting the study: (1) a theoretical framing is first outlined in terms of digital educational games and research on self- 
explanation, (2) methods employed in the study are outlined, (3) overall results are analyzed within and across experimental conditions in terms of the pre-test, post-test, engagement, and gameplay metrics, (4) results are analyzed within the adaptive self-explanation condition comparing performance across levels of abstraction within that condition, and (5) results, implications, caveats, and conclusions are discussed.

\section{Background}

Digital games are influential and ubiquitous presences in the lives of young learners. Digital games can be defined as interactive digital environments that (a) are based on a set of agreed rules and constraints, (b) are directed toward a clear goal that is often set as challenge, and (c) constantly provide feedback in terms of either scores or changes in the game world to allow players to monitor their progress toward the goal (Wouters, van Nimwegen, van Oostendorp, and van der Spek, 2013). A 2008 study by the Pew Internet and American Life Project found that $97 \%$ of teens aged 12-17 played computer, web, portable, or console games, and $50 \%$ of them reported daily or nearly daily play (Lenhart et al., 2008). A 2012 Pew study reported that digital games generated $\$ 25$ billion in sales in 2010 (Anderson \& Rainie, 2012).

With this massive interest in digital games, aspects of recreational games have spread into education. The current study defines "educational games" as games intended to support learning of academic content. The games could be referred to as "serious games" in the sense "that the objective of the computer game is not to entertain the player, which would be an added value, but to use the entertaining quality for training, education, health, public policy, and strategic communication objectives" (Wouters et al., 2013, p. 2). Investigation into the use of games for education has grown from a small niche area to a major focus of research over the past 15 years (e.g., Gee, 2007). Reports by the National Research Council and others (e.g., Authors, 2013; Honey \& Hilton, 2010; Young et al., 2012) have acknowledged this potential, but also 
acknowledged the unevenness of systematic evidence for games as learning tools. Furthermore, although several recent meta-analyses have demonstrated that games can successfully support learning, these reviews have underscored the importance of design choices to the efficacy of educational games (Authors, 2016b; Wouters et al., 2013). At the broadest level, the purpose of the current study is to explore a design approach for increasing the efficacy of digital educational games through the integration of research on self-explanation and learning.

\section{Self-Explanation and Learning}

Self-explanation can be defined as a content-relevant articulation expressed by the student as the student engages in a learning activity (Chi, 2000). Prompting for self-explanation can take many forms, including verbal prompts (Chi et al., 1994), prompts generated by computer tutors (Hausmann \& Chi, 2002; Aleven \& Koedinger, 2002), or promptsembedded in the actual learning materials (Hausmann \& VanLehn, 2007). The hypothesis underlying selfexplanation research is that prompting students to self-explain their actions and their thinking behind key steps in a learning activity causes higher learning gains than having students study the material without such prompting. Self-explanation is viewed as "a domain general constructive activity that engages students in active learning and insures that learners attended to the material in a meaningful way while effectively monitoring their evolving understanding" (Roy \& Chi, 2005, p. 272).

Research on self-explanation by Chi and others has explored the value of self-explanation for learning (e.g., Chi, Bassok, Lewis, Reimann, \& Glaser 1989; Roy \& Chi, 2005; Chi \& VanLehn, 1991).Roy and Chi's (2005) review of research on students' self-explanations reported that self-explanation resulted in average learning gains of $22 \%$ for learning from text, $44 \%$ for learning from diagrams, and 20\% for learning from multimedia presentations. According to Roy 
and Chi (2005), the process of self-explanation encourages four key cognitive mechanisms: (a) recognizing what information is missing while generating inferences,(b) integrating the information taught within the lesson, (c) integrating information from long term memory with new information, and (d) identifying as well as correcting incorrect information.Overall, selfexplanation can encourage students to engage in meta-cognitive activities to monitor what they do and do not understand.

\section{Self-Explanation and Educational Games}

Although research has shown that self-explanation can be effective for learning from texts, diagrams, and multimedia, research oneducational games hasdemonstrated fewer consistent benefits. While some studiessuggested efficacy in game-like settings (Moreno \& Mayer, 2005, Mayer \& Johnson, 2010, Johnson \& Mayer, 2010), other studies demonstratedthat self-explanation prompts can also result in either (a) extraneous processing by slowing down and distracting the playerfrom learning or (b) minimal processing due to learners ignoring the prompts in orderto return to gameplay as quickly as possible (Authors, 2014; O'Neil et al., 2014).

Research has attempted to explore the design structures and contextual conditions under which self-explanation functionality is effective within games. A series of experiments conducted by Moreno and Mayer (2005) with the simulation/game Design-a-Plant (Lester, Stone, \& Stelling, 1999) demonstrated that self-explanation facilitated additional learning only when the student is reflecting upon correct information and is not already engaged by the game in active cognitive processing. The first experiment showed no significant benefit for oral selfexplanations, possibly because the game's interactive elementsalready engaged learners cognitively.The second experiment showed an interaction between self-explanation and 
interactivity. Students in the non-interactive version performed better on transfer and retention tasks after engaging in self-explanation while students in the interactive version performed better on retention questions when they did not reflect on their answers.Moreno and Mayer proposed as an explanation that, because the answers were provided by the system, the non-interactive condition guaranteed that students were reflecting only on correct answers.Students in the interactive condition, however, were potentially reflecting on answers that were incorrect because they were generated by the students themselves.In a third experiment, interactive students and non-interactive students were asked to reflect on correct answers provided by the program or themselves. The results showed that performance on far-transfer tasks was best for the learners who reflected on the program's correct responses regardless of interactivity.This suggestedthat self-explanation is most effective when students are asked to think about correct solutions rather than general reflections.

One possible way to reduce the cognitive demands and potential distraction of selfexplanation prompts while ensuring that students are focusing on correct solutions involves providing learners with a set of explanation options.Using a game-like environment to teach about electric circuits, Mayer and Johnson (2010) found that students who were asked to select from a list of possible explanations to justify their answers performed significantly higher on the final transfer task level of the game (compared to students in the base version of the game). This result showed that even though the students did not generate the explanations themselves, adding the self-explanation questions prompted them to make connections between the game and the underlying rules of the electrical circuit system. The study also showed that there were no significant differences between students who received the self-explanation prompts, explanatory feedback, or a combination of the two.All three conditions facilitated performance on the final 
level of the game as well as improved performance, in terms of accuracy, on the first nine levels of the game.

In a second series of studies with the electric circuits games, Johnson and Mayer (2010) found that asking students to generate their own explanations did not result in significant improvement over the base version of the game.Students who selected an explanation from a list, however,showed significantly higher performance.Providing students with possible explanations as well as feedback can therefore decrease both incorrect thinking and extraneous processing.This result was fortunate from a pragmatic standpoint of design because open-ended self-explanation responses crafted by students may be difficult for game software to analyze in a responsive and feedback-rich manner.

Authors (2014) and O’Neil et al. (2014) have illustrated, however, that self-explanation questions may result in minimal processing even when students are provided with possible explanations.Authors (2014)highlighted the importance of minimizing the number of prompts and tightly coupling prompts with students' actions to optimize processing demands and to avoid disrupting the flow of the game. Authors (2014) and O’Neil et al. (2014) also noted that players sometimesactively avoided engaging in deeper processing during the self-explanation phase by randomly selecting each answer option until the game indicated that the correct answer has been selected. Thisdecision circumventedthe need to think or pay attention to the self-explanation functionality.Exploiting elements of the game interface to minimize thinking is known as "gaming the system" in a negative sense in the cognitive tutor literature (Baker et al., 2008).Authors' (2014) findings demonstrated that students showing higher circumventing behavior achievedsmaller learning gains regardless of learning condition.Hsu, Tsai, and Wang's (2012) study demonstrated a similar pattern. In Hsu et al.'s study, participants within the self- 
explanation group were divided into high- and low-engagement groups based on the ratio of correct and incorrect/“I don’t know” responses to self-explanation prompts. While there were no significant differences between Hsu et al.'s self-explanation and control conditions on the retention test overall, high-engagement students in the self-explanation condition scored significantly higher than the students in the control condition and the low-engagement students in the self-explanation condition. This suggested that educational game designers must design a balance that engages students in thinking deeply about the materialwithout overloading the students.

Lastly,designers ofself-explanation functionality for digital games must considerprompt abstraction.O’Neil et al. (2014) provided three different types of self-explanation questions designed to target different types of processing in their fraction game,Save Patch.The first type of questiontargetedessential/intrinsic processing and attemptedto attract the learner's attention to the units of distance that were used in the game. The second type of question was designed to help the learner draw connections between the game elements and the corresponding mathematical elements, thereby encouraging more intrinsic/essential processing. The thirdtype of question was intended to promote generative processing by asking the learner to think more abstractly about movement mechanics in the game environment.Students in the self-explanation group were given all three question types after each game level and were asked to make two answer selections from one or two of the questions.Although the students showed no difference in learning outcomes between the base game and the self-explanation group, the results showed that students within the self-explanation group who answered more questions that drew connections between the game mechanics and the math concepts (question type 2) showed better learning process scores on game features (e.g., fewer deaths or higher levels reached in the 
game). The authors noted that although abstract prompts were effective for older learners with the electric circuits game (Mayer \&Johnson, 2010; Johnson \&Mayer, 2010), younger children may find abstract rule statements too complicated.

O’Neil et al.'s (2014) results suggestedthat self-explanation prompts should not be too simple/concrete (question type 1) because students may only engage in minimal processing. O'Neil et al. also argued, however, that self-explanation prompts should not be too complex for the targetedlearners (question type 3) because that may result in extraneous processing. O'Neil et al.'s results also showed a significant correlation between gameplay measures and performance on the learning outcomes, suggesting that students' abilities to engage effectively in the game impacted their learning outcomes. Authors (2014) similarly proposed thatstudents in their study may have found the self-explanation prompts unhelpful because the prompts were too abstract. Authors (2014) also suggested that students are more likely to engage in deep processing when the self-explanation functionality focuses on students' specific challenges in the game.

While O’Neil et al. (2014) and Authors (2014)highlighted the challenges of incorporating self-explanation prompts that focus on abstract connections and relationships, these abstract connections and relationships are highly desirable in terms of learning goals. This tension raises the question of how a game design mightleverage and scaffold abstract connections through the self-explanation functionality without unduly increasing processing demands.

After Authors (2014), the first study exploring this line of research by our research group compared three conditionsfor providing high-abstraction self-explanation prompts (Authors, 2015d).Authors (2015d)compared three conditions: (a) explanatory feedback where the highabstraction relationship was provided directly to the student in the game dialog as the explanation for the navigation phase just completed, (b) high-abstraction self-explanation where 
the student selected self-explanation answers from a high-abstraction prompt for the navigationphase just created, and (c) full self-explanation where the student selected selfexplanation answersfor first a low-abstraction prompt, thena medium-abstraction prompt, and finallya high-abstraction prompt after the navigation phase.

Results from Authors (2015d) demonstrated that the high-abstraction self-explanation and full self-explanation conditionsdid not differ significantly. Therefore, there was a collapsing of the two conditions into a single "self-explanation" category for comparison with the explanatory-feedback condition. Overall, Authors (2015d) determined that the self-explanation conditions promoted better near-transfer learning outcomes than the explanatory-feedback condition, but only after controlling for the number of levels that students had completed. Additionally, students' inhibitory control abilities were correlated with learning outcomes forthe self-explanation condition but not for the explanatory feedback condition. Together, these results suggestedthat (a) self-explanation can benefit learning beyond explanatory feedback in educational games, but(b) the benefits of self-explanation are mediated by students' executive cognitive abilities. The findings underscored the challenges highlighted by O'Neil et al. (2014) and Authors (2014), including the increased processing demands inherent in adding the selfexplanation functionality as well as the danger of overloading students with the self-explanation functionality.

\section{Adaptivity in Educational Games}

The current study explores an approach to scaffold abstract connections through the selfexplanation functionality without unduly increasing processing demands by adaptively increasing the abstraction of self-explanation prompts as players increase in proficiency. Prior research indicates that adaptive feedback and prompts can improve learning. Chi et. al (2011) 
found, for example, that adaptive learning systems for teaching introductory college physics improved learners' learning gains significantly. Reif and Scott (1999) studied the differences between human tutoring, step-based tutoring, and no tutoring for a physics domain. They found that gain scores between human tutors and step-based tutors were not significantly different, but were significantly better than having no tutor. VanLehn (2011) found intelligent computerized tutors that adapted content according to learner performance outperformed human tutors. Atkinson, Renkl, \& Merrill (2003) demonstrated the efficacy of combined fading with the introduction of prompts designed to encourage learners to identify the underlying principle illustrated in each worked-out solution step.Azevedo, Cromley, \& Seibert (2004) found that students who used adaptive scaffolds to learn a complex science domainlearned better than students learning using fixed scaffolding or no scaffolding.

Research also shows that there are many feasible approaches to adaptive feedback. Ringenberg and VanLehn (2006), for example, found that adaptively providing students with worked out examples for physics problems was as effective as providing them with adaptive hints. Desmarais and Baker (2012) reviewed the learner models that have played the largest roles in the success of such learning environments and the latest advances in the modeling and assessment of learner skills. That said, different contexts afford different approaches, and some domains may be easier to support through adaptivity than others. Effect sizes for adaptive tutoring systems, for example, were the largest for STEM domains compared to humanities domains (Cohen, Kulik \& Kulik, 1982).

Adaptivity has been demonstrated as effective in digital games for learning. Oostendorp, van der Spek, and Linssen (2014) found that dynamically adapting the challenge level of a serious game about triage significantly increased learning among participants without affecting 
reported engagement. Sampayo-Vargas, Cope, He, and Byrne (2013) similarly found in a game focusing on Spanish cognates that adaptively adjusting the difficulty of the game led to significant increases in student learning without impacting engagement.Soflano, Connolly, and Hainey (2015)demonstrated the value of adaptivity within educational games in the sense that (a) learning styles identified using questionnaires were not always consistent with the learning styles demonstrated during gameplay and (b) learning styles fluctuated during the learning process in games. Lee, Rowe, Mott, and Lester (2014) and Rowe and Lester (2015) demonstrated supervised machine learning techniques (e.g., dynamic Bayesian networks) can effectively drive adaptation decisions to support significant improvements in student learning gains and problemsolving. Other research on adaptivity in educational games has found more mixed results. Conati and Manske (2009), for example, evaluated the impact of adaptive feedback on the effectiveness of a pedagogical agent in an educational computer game. They compared a version of the game with no agent to two versions with agents using different student models to guide the agent's interventions. Conati and Manske found no difference in student learning across the three conditions.

Digital games comprise a wide range of mechanics and formats and provide a wide range of options for integrating adaptivity as a result. Lopes and Bidarra (2011) surveyed adaptivity in digital games (both recreational games as well as serious games) and outlined several ways in which games can focus adaptivity: (1) the game world itself and the objects within it can be varied so that the world can be made easier or harder depending on proficiency, (2) the game mechanics in terms of how actions are implemented can be made easier or harder, (3) the attributes and algorithms of the nonplayer characters can be enhanced, (4) the sequence and pacing of the game narratives can be adjusted, and (5) game scenarios can be adapted within the 
narrative in terms of what a player encounters within a level of the game. The present study builds on existing research by exploring an approach within Lopes and Bidarra's fifth category of game scenarios in the sense of adaptively increasing the conceptual abstractness of selfexplanation prompts as a player's proficiency increases.

\section{The Current Study}

Based on the findings across the prior research on self-explanation in digital games discussed in the previous sections, six major design principles can be distilledconcerning effective integration of self-explanation functionality into educational games:

1. Students should be asked to reflect upon correct information.

2. To decrease intrinsic processing load and facilitate feedback, students should be provided with self-explanation "answers" from which to choose as opposed to being required to write open-ended responses.

3. Self-explanations prompts should take into account the intrinsic processing demands of the game itself, which may explain why self-explanation prompts have proven most effective in simpler game-like environments.

4. Self-explanation prompts should be tightly coupled with specific student actions and specific game challenges.

5. Students' levels of engagement can impact the efficacy of the self-explanation functionality.

6. The nature, focus, and abstractness of the self-explanation functionality can affect the students' levels of processing and learning outcomes.

In addition to these six design principles derived from prior research, the current study explores a seventh hypothesized design principle based on research on adaptive functionality. 
7. Adaptively adjusting the abstraction of self-explanation functionality might ameliorate the negative aspects of self-explanation functionality observed in prior research while supporting the positive aspects.

The current study explores this seventh hypothetical design principle by comparingthree conditions. The comparison is conducted in the context of self-explanation functionality designed in accordance with the first six design principles. In the navigation-only condition, players maneuver through navigational challenges without any self-explanation prompts. In the navigation+abstract condition, these navigational challenges are paired with a self-explanation prompt that focuses on abstract connections between the navigational challenges and Newtonian relationships. In the navigation+adaptive condition, the navigational challenges are paired with self-explanation prompts that adaptively increase from low abstraction (in which the prompts focus concretely on navigational moves)to high abstraction (in which the promptsfocus more abstractly on the navigational moves in terms of overarching Newtonian relationships). These three conditions were designed to test three core predictions:

1. Students in the navigation+adaptivecondition would demonstrate greater pretest-posttest gains than students in the navigation+abstract condition or the navigation-only condition.

2. Students in the navigation-only condition would progress significantly further in the game than the navigation+abstract condition because the navigation-only students would not require time to interact with the self-explanation functionality, the navigation-only students would not progress significantly further than the navigation+adaptive students because the adaptive design would make the self-explanation functionality less disruptive and more accessible. 
3. Students in the navigation+adaptive condition would display patterns in their gameplay behavior indicating deeper conceptual sophistication than the students in the other conditions.

\section{Methods}

\section{Participants}

The current study was conducted with the $7^{\text {th }}$ grade classrooms of two teachers in two different middle schoolsin the southeastern United States. A total of 210 students, including 124 from the first school and 86 from the second school, assented to participate in the study. Both schools were racially and culturally diverse. In terms of socio-economics, $43 \%$ of the students at the first school and $83 \%$ of the students at the second school were eligible for free or reduced lunch. Students were randomly assigned to one of three game conditions within each classroom (i.e., each classroom included students in all three conditions). Students were removed from the final data analyses if they did not complete the entire pretest and posttest or if their data were compromised in some other way. A total of 170 students were included in the final analyses, including 97students from the first school and 73 studentsfrom the second school.

The students represented a broad range of video game playing experience outside of school. In the survey administered to the students, students reported playing video games an average of 4.6 hours per week with a standard deviation of 3.7 and a range of 0 to 10 hours. These students therefore appear representative of the wide range of experience with digital games that students typically bring to the classroom.

Five days were spent in each school constitutingone week of class time. In all classes, students were instructed on how to navigate through initial steps in the game and received help as needed. Students were also encouraged to talk about the game and share strategies with their 
peers if they got stuck. Thirty minutes before the end of the last class on the final day, students were asked to stop playingand were guided through the post-test and a short engagement survey. Because experimental procedures were similar across schools and students were assigned across all conditions in each classroom, students were aggregated across classrooms and schools for the analyses.

\section{The Fuzzy Chronicles Game and Experimental Conditions}

Fuzzy Chronicles is a digital game that was developed to support students learning about Newtonian dynamics (Authors, 2012a; Authors, 2015a; Authors, 2016). Students play as the space navigator Surge who must pilota spaceship through a two-dimensional spatial grid (see Figures 1 and 2) by placing actions at waypoints along a trajectory. Game play is divided into levels, each constituting a separate navigational challenge. Overall, players complete levels of the game that require them to consider the appropriate magnitude and direction of forces to propel their ships in a desired direction at a desired speed. Speed gatesin a level require the ship to travel at a specific speed to pass through safely (Figure 1). A player may also pick up fuzzies, which add mass to her/his ship. Mass gatesallowonly ships with a certain amount of mass to pass through safely. Players may also throw fuzzies. Throwing a fuzzy launches the fuzzy in one direction while applying an equal and opposite force to the ship.

Players create a flight plan by placing actions on the map at waypoints (Figure 2). Actions include forces of various magnitudes and directions as well as other commands to pick up, release, or throw fuzzies. When the player is ready, she/he clicks the launch lever. Surge's ship is then launched. Actions are executed if and when Surge's ship passes through the waypoint where the actions were placed. The plan may or may not be successful, and actions may or may not be executed depending on whether the ship actually reaches the waypoints 
where the actions wereplaced. If the player is successful, the next level in the game is unlocked and she/hecan proceed. If the user is not successful, the player returns to the planning phase in order to add, change, move, or delete any of the actions. Therefore, players cannot skip ahead. [Insert Figures 1 and 2 Here]

Players navigate from one level to the next on a star map that contains differently colored regions designating progressively more challenging combinations of Newtonian concepts. This study focuses on the first two regions, red and blue, because those are the regions that most students can feasibly complete in one week of class time. Levels in the red region focus on relationships between force and changes in velocity. Players alter the direction and magnitudes of forces on their shipsin order to manipulate their speed and direction (i.e., velocity) across the map to reach the exit portal. Levels in the blue region focus on relationships between force, mass, and changes in velocity. Blue levels are similar to red levels with the addition of an emphasis on mass. Players pick up fuzziesalong their trajectory. Fuzzies have a mass of 1 kilogram each andplayers mustadjust their force calculations to account for their increased mass.

The red and blue regions each include four or five introductory levels, which introduce the new ideas and mechanics of the region, as well as two boss levels at the end, which are more challenging and require the player to integrate across the mechanics and ideas of the region. In addition to the introductory levels and boss levels in a region, a warpmission (Figure 3) is included in each region to separate the introductory levels for the region from the culminating challenge, or boss, levels for the region.

\section{[Insert Figure 3 Here]}

The warp mission, which is located in between the introductory and boss levels, is intended to support the player in mastering the basic ideas of the region before progressing to the 
boss levels of that region (Figure 3). Warp mission levels differ from introductory and boss levels in several important ways. First, warp mission levels involve multiple variants of the mission that must be completed before a player can unlock and progress to the boss levels.More specifically, missions for a warp mission level must be completed multiple times until the player has raised her/his overall average mission score for that level from a beginning score of 0 to a score of 80 , which indicates a high level of proficiency. Players receive a new, but similar, variant of the warp mission on each attempt. Warp mission levels are thus different from introductory and boss levels because introductory and boss levels (a) need to be successfully completed only once to unlock the subsequent level and (b) do not change from one attempt to the next.

Essentially, each time the player attempts a warp mission level, the player receives one of several variants of the mission that involve the same conceptual and navigational challenge but a different layout and orientation. The player's score for the variant is based on the number of attempts needed to solve that variantand its associated explanation prompts, with more attempts resulting in a lower score. The new overall score for the ongoing warp mission level is the average of the player's score on the most recent variantand the player's current overall score for the level. In this way, the overall score for a warp mission level is essentially a running average of scores on the variants of that warp mission level.Students therefore (a) repeatedly encountervariants of the mission until the running average of those scores exceeds a certain threshold and (b) receive feedback on their answers to the self-explanation prompts.

The current study compares three game conditions. The researchers hereafter refer to these conditions simply as nav+adaptive, nav+abstract, and nav-only for brevity. These conditions differed only in terms of the type of the self-explanation functionality, if any, included 
in thewarp mission levels. As described earlier, the nav-only condition does not pose selfexplanation prompts after the navigation phase. Nav+abstract and nav+adaptive conditions pose self-explanation prompts targeted at the concepts just encountered in the navigation phase. In the nav+adaptive condition, these explanation prompts begin as "low abstraction" and then increase to "medium abstraction" and eventually to "high abstraction" as the player's running average of scores for that level increases. Low-abstraction prompts and answers focus on articulating the concrete specific actions that the student chooses in the navigation phase. An example of a lowabstraction prompt and answers would include:

Prompt: What force did you exert for 0.1 seconds to speed up from $2 \mathrm{~m} / \mathrm{s}$ to $4 \mathrm{~m} / \mathrm{s}$ ?

Answer: I exerted a $20 \mathrm{~N}$ force to speed up by $2 \mathrm{~m} / \mathrm{s}$.

High-abstraction prompts and answers focus on underlying rules and relationships. An example of a high-abstraction prompt would include:

Prompt: How does exerting a force change the speed of the ship?

Answer: The more force there is, the more the ship's speed will change. If you multiply the force, the change in speed multiplies by the same number.

In the nav+abstract condition, the self-explanation prompts are always "high abstraction."

\section{Physics Assessment}

A twenty-one question, multiple choicepre-post test was created to assess physics understanding. The pre-test and post-test were identical. Test questions asked students to determine changes in velocity from the application of various forces as well as to determine the magnitude and direction of forces required to achieve various changes in velocity (Figure 4 presents an example question). 
A Cronbach alpha analysis of test item reliability demonstrated good levels of reliability for the twenty-one items combined (0.834). Individual proposed subsets of questions also demonstrated fair reliability, with questions aboutforce and velocity change focal to the Red levels scoring 0.716 and questions about mass relationships focal to the Blue levels scoring 0.738.Item difficulties were estimated for each of the questions with a two-parameter item response theory model using the Itm package in R (Rizopoulos, 2006).A third classroom implementation included six of the questions, and those data were also included for this analysis. In an item response framework, item difficulties were calculated on a latent scale with a practical range of -4 to 4 , where an estimated difficulty of 0 indicates an average level of difficulty for the sample population.Based on the estimated item difficulties for these runs, it was determined that the average difficulty estimate for the 7 blue mission items (1.79) was higher than the average difficulty estimate of the 7 red mission items (1.48). This finding was not surprising because blue mission questions deal with mass in addition to the concepts present in the red missions. A differential item functioning (DIF) analysis of all post-test items was conducted, and it found no significant difference in item functioning across each of the three experimental conditions.

\section{Results}

Results are first presented within and across experimental conditions in terms of the pretest, post-test, engagement, and gameplay metrics. Results are then presented within the adaptive condition comparingperformance on the low-abstraction, medium-abstraction, and highabstraction explanation prompts.

\section{Pre-Test and Post-Test Scores.}

A one-way ANOVA of pre-test scores was conducted for the three conditions (Table 1). As expected, there were no significant differences between the three conditions on the pre-test 
$(\mathrm{F}(2,132)=2.248, \mathrm{p}=0.110)$. Next, a one-way ANOVA of post-test scores was conducted. The one-way ANOVAshowed that the difference in post-test scores between nav-only, nav+abstract, and nav+adaptive conditions was significant, $\mathrm{F}(2,132)=3.215, \mathrm{p}=.043$. Tukey's HSD test showed that the post-test scores of students in the nav+adaptive condition were significantly higher than those of the students in the nav+abstract condition.

Paired t-tests between pretest and post-test scores for each condition at each school were then run (Table 2). All of these paired t-tests showed significant improvement $(p<0.001)$ from pretest to post-test (nav-only: $\mathrm{t}(43)=-8.898$, nav+adaptive: $\mathrm{t}(37)=-6.832$, nav+abstract: $\mathrm{t}(52)=$ -6.568). The effect sizes for each condition were calculated using Cohen's d corrected for paired T-tests (nav-only: 1.309, nav+adaptive: 1.151, nav+abstract: 0.904). These findings demonstrated that students in all conditions scored significantly higher on the post-test than the pre-test with a large effect size.

[Insert Tables 1 and 2 Here]

\section{Highest Level Completed}

ANOVAs were conducted to compare the experimental conditions for progress in the game (Table 1).Students could move onward to a subsequent game level only after successfully completing the game level preceding it. For this reason, the highest game level a student completed measured how far the student progressed in the game. A one-way ANOVA showed that the difference in highest level completed between nav-only, nav+abstract, and nav+adaptive conditions was statistically significant, $F(2,132)=10.658, \mathrm{p}<0.001$. Tukey's HSD test showed that the highest level completed was significantly higher in the nav+adaptive and nav-only conditions than in the nav + abstractcondition.

\section{Engagement}


Students completed an engagement survey where they responded to four statements that would indicate their level of engagement in the game on a scale of $1=$ Strongly Disagree to $5=$ Strongly Agree.Students' scores fortheir surveys were summed and reported here as engagement (Table 1).Cronbach Alpha for the four items was .875.A one-way ANOVA of engagement among the three conditions found that there was no significant difference in engagement across the three conditions, $F(2,132)=0.465, \mathrm{p}=0.629$.

[Insert Tables 3 and 4 Here]

Pearson correlations between total engagement and post-test score and between total engagement and highest level completed for students in each condition were conducted (Tables 3 and 4). None of the correlations between engagement and post-test score were significant (navonly: $\mathrm{r}=-0.142, \mathrm{p}=0.357$; nav+abstract: $\mathrm{r}=-0.233, \mathrm{p}=0.093$; nav+adaptive: $\mathrm{r}=-0.196, \mathrm{p}=$ 0.237). This suggeststhat student engagement was not related to post-test score in any condition. Total engagement, however, was significantly negatively correlated with highest level completed in the nav+abstract $(\mathrm{r}=-0.402, \mathrm{p}=0.003)$ and nav+adaptive $(\mathrm{r}=-0.693, \mathrm{p}<0.001)$ conditions, and the correlation was nearly significant in the nav-only condition $(r=-0.276, p=0.07)$. This suggests that engagement as self-reported by the students was negatively correlated with their highest level completed, particularly for the conditions with the explanation prompts.

\section{Actions Used and Attempts Per Level}

Next, two gameplay performance metrics were examined: (a) how many changes students made to their navigation plans on each attempt of a level on average and (b) the average number of attempts used per level completed (i.e., total attempts divided by the total number of levels completed in the game). These metrics provided insight into the degree to which players thought 
about or understood the underlying relationships in the game(i.e., as opposed to guessing, bruteforcing, or iteratively nudging their navigation plans toward successful configurations).

Average actionsper attemptwerecalculated as the average number of additions, deletions, or changes a player made to her/his navigation plan each time the player attempted or reattempted a game level. A one-way ANOVA showed that the difference in actions used per attempt between nav-only, nav+abstract, and nav+adaptive conditions was statistically significant, $F(2,132)=42.009, \mathrm{p}<0.001$. Tukey's HSD test showed that students in the nav+adaptive and nav + abstract conditions used significantly more actions for each attempton average than those in the nav-only condition.

Average attempts per level werecalculated as the total number of attempts divided by the highest level in the game successfully completed by a player. A smaller average meant that a player attempted most levels only a few times before succeeding, a higher average number of attempts meant that the player attempted each level more frequently. A one-way ANOVA showed that the difference in the average number of attempts needed to complete each non-warp level between nav-only, nav+abstract, and nav+adaptive conditions was not statistically significant, $\mathrm{F}(2,132)=1.305, \mathrm{p}=.275$.

\section{Abstraction of Prompts Within the Nav+Adaptive Condition}

Finally, student scores were analyzed within the nav+adaptive condition on the lowabstraction, medium-abstraction, and high-abstraction self-explanation prompts to compare student performance across the levels of abstraction (Table 5).Repeated measures ANOVA(F(2, $115)=10.153, p=0.002$ ) showed that students scored significantly differently across levels of abstraction. This difference was significant for the comparison of high versus low and the comparison of high versus medium, using Bonferroni's correction for multiple comparisons, 
indicating that students performed better on the high-abstraction prompts than the less abstract ones (High vs. Medium $=9.291, \mathrm{p}=0.001$; High vs. Low $=5.787, \mathrm{p}<0.001)$. There was no significant difference between the scores for the low-abstraction and medium-abstraction prompts (Medium vs. Low $=-3.505, \mathrm{p}=0.358)$.

\section{[Insert Table 5 Here]}

\section{Discussion}

Students improved significantly from pretest to posttest with a large effect size in all conditions. Prior research demonstrated thattaking the pre-test by itself does not lead to increased outcomes on the post-test(Authors, 2015c; Authors, submitted). Theincreased post-test scores can therefore be interpreted as representing learning from the game as opposed to merely a testing effect.

It was anticipated thatthe nav+adaptive condition, in which students received selfexplanation prompts that adaptively increased in abstraction,woulddemonstrate significantly higher post-test scores than the nav+abstract and nav-only conditions, in which students respectively received only high-abstraction self-explanation prompts or no self-explanation prompts. While the post-test results of the nav+adaptive condition were indeed higher than the results of the nav-only and nav+abstract conditions, this difference was significant only for the nav+abstract condition. This indicates that while the nav+adaptive approach was a significant improvement over the nav+abstract approach, the nav+adaptive was not significantly more effective than the nav-only approach in terms of the post-test results.

Student progression through the game might explain some portion of these patterns.It was expected that (a) the nav-only group would complete significantly more levels in the game than the nav+adaptive or nav+abstract groups because the nav-only group would not have any self- 
explanation prompts to complete along the way and (b) the nav+adaptive group would complete significantly more levels in the game than the nav+abstract group because the adaptive approach would make the self-explanation prompts more accessible for the nav+adaptive group.These hypotheses proved correct, but only the difference between nav-only and nav+abstract and the difference between nav+adaptive and nav+abstract were significant. While the nav-only students completed more levels in the game than the nav+adaptive students, this difference was not significant, suggesting that the time required for the adaptive self-explanation functionality was partially offset by time saved by the nav+adaptive students in the rest of the game.

Perhaps the adaptive self-explanation functionality bolstered learning enough for students to glean useful content insights that allowed them to progress faster through the game. Alternatively, perhaps the adaptive nature of the nav+adaptive condition allowed students to move more quickly through the warp missions, thereby allowing them more time to work on other levels in a manner similar to the advantages of the nav-only condition. Future research should explore these alternatives to understand the underlying relative contributions and mechanisms. Current findings make clear, however, that thenav+adaptive students progressed significantly further than the nav+abstract students. This progress, although not as extensive as the progress of the nav-only students, combined with whatever other pedagogical advantages of the adaptive prompts to support significantly higher performance on post-tests for the nav+adaptive students relative to the nav+abstract students. Relative to the nav-only students, the nav+adaptive students may have scored higher but not significantly higherbecause the nav-only students'somewhatfurther progress in the game diluted some of the pedagogical advantage of the self-explanation functionality in terms of the post-test. 
Turning to engagement, it was predicted that students in the nav-only condition would report the highest levels of engagement on the survey because it wasexpected that the selfexplanation prompts of the other two conditions would distract from game play. Additionally, it was also expected that the nav+adaptive condition would report higher engagement than the navabstract conditionbecause it was assumed that the adaptation of the self-explanation prompts in the nav+adaptive condition would causethe self-explanation functionality to flow more smoothly and naturally for students. Despite these predictions, there were no significant differences in total engagement across conditions. This represents progress from the studies with earlier designs of Fuzzy Chronicles, where the designs of the self-explanation prompts were too intrusive and disrupted gameplay (e.g., Authors, 2014). These findings support the design decisions underpinning the current version of the game: (a) focusing the self-explanation prompts primarily in the warp missions rather than including self-explanation prompts with every level, (b) presenting only one self-explanation prompt after each warp mission navigation phase, (c) creating short and focused navigation phases for each warp mission variant so that the connection between the self-explanation prompt and the navigation phase was clear and obvious, (d) presenting the self-explanation prompt only after the successful completion of the navigation phase so that students reflectedonly on successful solutions and remained undisrupted in their gameplay, and (e) integrating the self-explanation prompts more deeply into the progression mechanics of the game such that students could not ignore the prompts by randomly clicking on responses.

Exploring the role of engagement more deeply, there were no significant correlations between engagement and post-test score for any condition, but there were significant negative correlations (for nav+adaptive and nav+abstract), and a trend toward significant negative 
correlation (for nav-only), between engagement and highest level completed. This indicatesthat as engagement increased, the highest level completed in the game decreased. This seems counterintuitive at first, but might suggest that the students who progressed the furthest through the game tended to be those who were the most focused on the game as a school assignment to maximize.

In terms of other game performance metrics, the average number of actions used per attempt and the average number of attempts per completed game level were counted. It was expected that the nav+adaptive conditionwould utilize significantly more actions per attempt but fewerattempts per level than the nav+abstract or nav-only conditions, and it was anticipatedthat the nav+abstract condition would utilize significantly more actions and fewer attempts than the nav-only condition. It was assumed that students who were conceptually best prepared for the game, which washypothesizedto be the nav+adaptive students, would be able to engage in more sophisticated planning for each attempt. It was also anticipated that those students would use a higher number of actions per attempt, and that their navigation plans would also be more successful, ultimately requiring fewer attempts per completed level.

These hypothesized differences parallel the distinctions between model-based reasoning and constraint-based thinking that Parnafes and diSessa (2004) observed in students working with a game-like environment called NumberSpeed. Students exercising constraint-based thinkingdo not try to understand and predict the whole system of relationships in a challenge. Instead, they simply make a few modifications, observe the outcomes of these local modifications, and then make a few more modifications in response. In this way, they "nudge" the plan closer toward a successful solution. Model-based reasoning, however, involves considering the underlying relationships of the challenges in a deep and comprehensive way in 
order to plan a solution that accounts for those relationships and addresses the challenge more globally as a system. This distinction in strategies is frequently observed inclassrooms using SURGE games. It was therefore predicted that the students in the nav+adaptive condition would evidencehigher degrees of model-based thinking because it was hypothesized that the nav+adaptive condition would support a deeper understanding of the underlying relationships. It was anticipated that the nav+adaptive condition students would therefore (a) demonstrate higher number of actions used per attemptbecause students would design more complete solutions as opposed to piecemeal nudging and (b) demonstrate fewer attempts on average per level because of the relatively deeper understanding involvedin the nav+adaptive condition compared to the other two conditions.

The findings supported the prediction concerningthe average number of actions used per attempt.Thenav+adaptive and nav+abstract students utilized a significantly greater number of actions per attempt than the nav-only students, and there was no significant difference in the number of actions used between nav+adaptive and nav+abstract. In terms of attempts per level, the nav+adaptive condition did have the lowest number of attempts per level, but the differences across conditions were not significant.

Finally, student warp mission scores were analyzed within the nav+adaptive condition on the low-abstraction, medium-abstraction, and high-abstraction self-explanation prompts. O’Neil et al. (2014) and Authors (2014) demonstrated the challenges of incorporating self-explanation prompts that focus on abstract connections and relationships. O'Neil et al. (2014), in particular, uncovered important relationships concerning learning outcomes and the types of prompts with which students choose to engage. Specifically, O’Neil and colleagues argued that selfexplanation prompts should not be too simple lest students engage only in minimal processing, 
nor too abstract as to cause confusion and extraneous processing. Thus, abstraction was desirable albeit with the caveat of potentially overwhelming students.

In interpreting the findings for this study, it is important to remember that students first needed to master the low-abstraction prompts before encountering the medium-abstraction prompts and, likewise, needed to master the medium-abstractionprompts before encountering the high-abstractionprompts. It is also important to remember that the navigation phases accompanying each self-explanation prompt were isomorphic for the low, medium, and highabstractionprompts.As stated previously, the scorefor a warp mission variant is the average of the scores on the navigation and explanation phases of that mission variant.

The paired t-tests demonstrate that students in the nav+adaptive condition earned significantly higher scores on variants with high-abstraction prompts than onvariants with medium-abstraction orlow-abstractionprompts. This was unexpected because it was anticipated that prompts with higher levels of abstraction would be more difficult than prompts with lower levels of abstraction (e.g., O’Neil et al., 2014). Specifically, it was expected that the lowabstraction scores would be significantly higher than the medium-abstraction scores, and that the low and medium abstraction scores would be significantly higher than the highabstractionscores. There are, however, several possible explanations for the results.

An optimistic explanation might be that the less abstract prompts effectively scaffolded students' understandings for the more abstract prompts. From this perspective, students were prepared for the high-abstractionvariants by the time they reached them. A more general explanation, however, might be that students also became better at answering promptsin general. A third explanation might be that students improved on the navigation components of thevariants, which also influenced score. All three of these factors likely contribute. Regardless 
of the relative contributions, however, students' higher scores on the high-abstraction variants suggest that adaptively increasing the abstraction of the self-explanation prompts was an effective approach to providing high-abstraction prompts in an accessible manner.

\section{Caveats and Limitations}

It is reasonable to argue whether or not the self-explanation functionality in the current study should be consideredsolely as self-explanation functionality. As described, students needed to achieve a certain score on the warp mission before being allowed to move on to the subsequent challenge levels for that section of the game. The goals involved (a) minimizing incentives for "gaming the system" in a negative sense to circumvent the self-explanation functionality (Baker et al, 2008) and (b) integrating the self-explanation prompts more fully into the "game atoms" and structures of the game itself rather than inserting them awkwardly outside the "atoms" of the game as an artificial appendage (Echeverria, Barrios, Nussbaum, Amestica, \& Leclerc, 2012).

For this study, each time a player attempted a warp mission, she/he received one of several variants of that mission that involved the same challenge conceptually and navigationally but in a different layout and orientation. The overall score for the warp mission levelwas the running average of the player's most recent score on the most recent variant and the player's previous overall score for the warp mission level prior to that variant (i.e., essentially a running average for that warp mission level). Students therefore (a) repeatedly encountered variants of the mission until the running average of their variant scores exceeded a certain threshold and (b) received feedback on their answers to the prompts. This is very different from Chi's initial approach to self-explanation, in which students received no feedback and were merely encouragedto answer the prompt with some form of explanation (e.g., Chi et al., 1994). 
It wasdecided to integrate the self-explanation functionality in the current study into coherent game atoms such that students could not simply ignore the prompts by randomly clicking on responses.Following from the findings of relevant research,additional principles in the design of the self-explanation functionality were added:(a) confining the self-explanation prompts to the warp missions rather than including self-explanation prompts with every level, (b) presenting only one self-explanation prompt after each warp mission navigation phase, (c) creating short and focused navigation phases for each warp mission variant so that the connection between the self-explanation prompt and the navigation phase is clear and obvious, and (d) presenting the self-explanation prompt only after the successful completion of the navigation phase so that students reflect on only successful solutions and do not have their gameplay disrupted.In this manner, the approach builds upon the evolving research on integrating self-explanation prompts into digital games. It is possible, however, that new terminology is needed to differentiate the approach from the original intended meaning of selfexplanation.

A second question to consider involves the potential generalizability of the approach. Many game genres are reported in the literature, such as action, puzzle, role playing, and many others. Each game genre has different game elements and functionalities. Furthermore, the disciplinary focus in the current study, Newtonian mechanics, is only one of a huge range of possible foci. The self-explanation functionality approach explored in the current study is only of value to the extent that it generalizes to some extent across some subset of games genres and/or disciplinary topics. In terms of game genres, generalizability appears promising mechanically because the approach is relatively modular; it focuses on the addition of dialogue interactions after key short levels in the game progression. Therefore, the approach is mechanically feasible 
across many genres including action, puzzle, role-playing, and others. The much bigger challenge for implementation, however, involves integrating these dialogue interactions in a manner that does not disrupt the flow of gameplay for the player. As outlined earlier, research on Fuzzy Chronicles with an earlier version of the self-explanation functionality by Authors (2014)demonstrated that the design of the self-explanation functionality in that earlier version was too overbearing and intrusive in terms of its timing, frequency, and structure. As a result, that version of the self-explanation functionality disrupted gameplay flow, gameplay progress, and learning. Thus generalizability across genres is mechanically very feasible, but requires careful design in terms of its structure, timing, and frequency as well as its narrative integration.

The second aspect of generalizability involves generalizability across disciplinary topics and the learning goals the game designers intend for the game. The self-explanation functionality explored in the current study and in previous research (e.g., Moreno \& Mayer, 2005; Hsu, Tsai, \& Wang, 2012) assumes that the learning goals for the game involve having the players come to understand and articulate relationships that they are exploring through their gameplay. Furthermore, the self-explanation approach outlined assumes that the designers can articulate normative and non-normative relationships that players are likely to perceive so that appropriate dialogue prompts and options can be crafted within the system such that players will find the dialogue options relevant and meaningful in terms of their own perceptions as they reflect on their gameplay. Thus in terms of disciplinary topics, the self-explanation approach outlined would appear to generalize beyond Newtonian mechanics to other domains that are wellstructured in terms of their underlying causal, logical, or mathematical relationships, but further research would be required to explore generalizability to less structured domains or less structured learning goals. 


\section{Conclusion}

Students in all conditions improved from pre-test to post-test with a large effect size. This suggests that the overall design of the Fuzzy Chronicles game itself is improving over the development and refinement iterations compared to earlier studies and pilot studies with Fuzzy Chronicles(Authors, 2014; Authors, 2015a). At the most basic level, this outcome demonstrates the importance of design beyond medium in developing digital games for learning as highlighted in an earlier meta-analysis of digital games, design, and learning (Authors, 2016b). In addition to overall refinement of level and concept sequences, control schemes, and user interface, the current version of Fuzzy Chronicles instantiated the features demonstrated as critical for selfexplanation functionality in games based on the findings of prior research on games and selfexplanation (e.g., Authors, 2014; Moreno \& Mayer, 2005; Mayer \& Johnson, 2010; Johnson \& Mayer, 2010).

In addition, the current version of Fuzzy Chroniclesintegrated theself-explanation prompts into the structure of the game such that they affected score and progress in the game. As discussed in the Caveats and Limitations section, this structure arguably transforms the functionality beyond the original conceptions of research on self-explanation (Chi et al., 1989, Chi et al. 1994). Thefunctionality addresses two concerns, however, by(a) minimizing incentives for "gaming the system" in a negative sense to circumvent the self-explanation functionality (Baker et al, 2008) and (b) integrating the self-explanation more fully into the "game atoms" and structures of the game itself rather than inserting them awkwardly outside the "atoms" of the game as an artificial appendage (Echeverria, Barrios, Nussbaum, Amestica, \& Leclerc, 2012). Importantly, the engagement survey demonstrated no significant overall differences across conditions, which suggests relatively successful integration from that perspective. 
In terms of the focal research question, an approach to adaptively increasing the abstraction of the self-explanation prompts encountered by students in the nav+adaptive condition was explored. As discussed, the high-abstraction prompts focused on key Newtonian relationships underlying gameplay, while the low-abstraction prompts focused concretely on the student's actions that led to a successful solution of the specific navigational challenge just completed. The findings demonstrate that this approach resulted in significantly higher post-test scores in the nav+adaptive condition relative to the nav+abstract condition, which included the same number of prompts overall. Furthermore, the analyses of students' scores in the nav+adaptive condition show significantly higher scores on average on the high-abstraction prompts than on the low or medium-abstraction prompts. This suggests that the adaptive structure, beginning with low-abstraction prompts and then increasing abstraction as student readiness increases, effectively scaffolds students to mastering the high-abstraction prompts.

Authors (2014) and O’Neil et al. (2014) concluded that, along with generative processing, self-explanation prompts can result in (a) extraneous processing by slowing down the player and distracting them from learning or (b) minimal processing if learners ignore the prompts and return to gameplay as quickly as possible. O’Neil et al. further concluded that high levels of abstraction in the self-explanation functionality may increase processing demands too substantially. The adaptive features: (a) resulted in significantly greater post-test scores than thenon-adaptive high-abstraction functionality, (b) scaffolded students smoothly up to the highabstraction prompts by focusing on the underlying Newtonian relationships, and (c) may have minimizedadverse impact of self-explanation prompts in a manner allowing students to progress significantly further in the game than students in the non-adaptive nav+abstract condition. 
While the post-test results of the nav+adaptive condition were higher than in the nav-only and nav + abstract conditions, this difference was significant only with regard to the nav+abstract condition. Thus, while the nav+adaptive approach was a significant improvement over the nav+abstract approach, the nav+adaptive was not significantly better than the nav-only approach in terms of the post-test results. This might be connected to the finding that the nav-only students still progressed further, although not significantly further, in the game than the nav+adaptive condition.

Looking beyond the post-test scores, however, the nav+adaptive and nav+abstract conditions elicited different game play behaviors than the nav-only condition. Specifically, students in the conditions with the self-explanation functionality used a higher average number of actions per attempt than did students in the nav-only condition. This suggests that the students in the conditions with the self-explanation functionality may have engaged in higher degrees of model-based thinking than the students in the nav-only condition (Parnafes \& diSessa, 2004). If the self-explanation functionality is indeed fostering higher-degrees of model-based thinking, it might be assumed that the self-explanation functionality is providing additional advantages uncaptured by the current post-test format. 


\section{References}

(Authors, 2012a)

(Authors, 2012b)

(Authors, 2014)

(Authors, 2015a)

(Authors, 2016b)

(Authors, 2015c)

(Authors, 2015d)

(Authors, 2016)

(Authors, 2013)

(Authors, submitted)

Aleven, V. A., \& Koedinger, K. R. (2002). An effective metacognitive strategy: Learning by doing and explaining with a computer-based Cognitive Tutor. Cognitive science, 26(2), 147-179.

Anderson, J. \& Rainie, L. (2012). Gamification: Experts expect 'game layers' to expand in the future, with positive and negative results.Pew Internet \& American Life Project. Available at: http://www.pewinternet.org/2012/05/18/the-future-of-gamification/

Atkinson, R. K., Renkl, A., \& Merrill, M. M. (2003). Transitioning From Studying Examples to Solving Problems: Effects of Self-Explanation Prompts and Fading Worked-Out Steps. Journal of Educational Psychology,95(4), 774. 
Azevedo, R., Cromley, J. G., \& Seibert, D. (2004). Does adaptive scaffolding facilitate students' ability to regulate their learning with hypermedia?. Contemporary Educational Psychology, 29(3), 344-370.

Baker, R., Walonoski, J., Heffernan, N., Roll, I., Corbett, A., \& Koedinger, K. (2008). Why students engage in gaming the system behavior in interactive learning environments. Journal of Interactive Learning Research, 19(2), 185-224.

Chi, M. T. (2000). Self-explaining expository texts: The dual processes of generating inferences and repairing mental models. Advances in Instructional Psychology, 5, 161-238.

Chi, M. T. H., \& VanLehn, K. A. (1991). The content of physics self-explanations. Journal of the Learning Sciences, 1(1): 69-105

Chi, M. T. H., Bassok, M., Lewis, M. W., Reimann, P., \& Glaser, R. (1989). Self-explanations: How students study and use examples in learning to solve problems. Cognitive Science, 13(2), 145-182.

Chi, M. T. H., DeLeeuw, N., Chiu, M., \& LaVancher, C. (1994). Eliciting self-explanations improves understanding. Cognitive Science, 18, 439-477.

Chi, M., VanLehn, K., Litman, D., \& Jordan, P. (2011). Empirically evaluating the application of reinforcement learning to the induction of effective and adaptive pedagogical strategies. User Modeling and User-Adapted Interaction, 21(1-2), 137-180.

Cohen, P. A., Kulik, J. A., \& Kulik, C. L. C. (1982). Educational outcomes of tutoring: A metaanalysis of findings. American Educational Research Journal,19(2), 237-248. 
Conati, C., \& Manske, M. (2009, September). Evaluating adaptive feedback in an educational computer game. In International Workshop on Intelligent Virtual Agents (pp. 146-158). Springer Berlin Heidelberg.

Desmarais, M. C., \& d Baker, R. S. (2012). A review of recent advances in learner and skill modeling in intelligent learning environments. User Modeling and User-Adapted Interaction, 22(1-2), 9-38.

Dimitris Rizopoulos (2006). Itm: An R package for Latent Variable Modelling and Item Response Theory Analyses, Journal of Statistical Software, 17(5), 1-25. URL http://www.jstatsoft.org/v17/i05/

Echeverria, A., Barrios, E., Nussbaum, M., Amestica, M., \& Leclerc, S. (2012). The atomic intrinsic integration approach: A structured methodology for the design of games for the conceptual understanding of physics. Computers \& Education, 59(2), 806-816.

Gee, J. P. (2007). What video games have to teach us about learning and literacy (2nd ed.). New York, NY: Palgrave Macmillan.

Hausmann, R. G., \& Chi, M. H. (2002). Can a computer interface support selfexplaining. Cognitive Technology, 7(1), 4-14.

Hausmann, R. G., \& Vanlehn, K. (2007). Explaining self-explaining: A contrast between content and generation. Frontiers in Artificial Intelligence and Applications, 158, 417.

Honey, M. A., \& Hilton, M. (Eds.). (2010). Learning science through computer games and simulations. National Research Council. Washington, DC: National Academy Press. 
Hsu, C.-Y., Tsai, C.-C., Wang, H.Y. (2012). Facilitating third graders' acquisition of scientific concepts through digital game-based learning: The effects of self-explanation principles. The Asia-Pacific Education Researcher, 21(1), 71-82.

Johnson, C.I., \& Mayer, R.E. (2010). Applying the self-explanation principle to multimedia learning in a computer-based game-like environment. Computers in Human Behavior, 26, $1246-1252$.

Lee, S. Y., Rowe, J. P., Mott, B. W., \& Lester, J. C. (2014). A supervised learning framework for modeling director agent strategies in educational interactive narrative. IEEE Transactions on Computational Intelligence and AI in Games, 6(2), 203-215.

Lenhart, A., Kahne, J., Middaugh, E., Macgill, A., Evans, C., \& Vitak, J. (2008). Teens, video games, and civics (p. 64). Pew Internet \& American Life Project. Retrieved from http://www.pewinternet.org/Reports/2008/Teens-Video-Games-and-Civics.aspx

Lester, J. C., Stone, B., \& Stelling, G. (1999). Lifelike pedagogical agents for mixed-initiative problem solving in constructivist learning environments. User Modeling and UserAdapted Interaction, 9, 1-44.

Lopes, R., \& Bidarra, R. (2011). Adaptivity challenges in games and simulations: a survey. IEEE Transactions on Computational Intelligence and AI in Games, 3(2), 85-99.

Mayer, R. E., \& Johnson, C. I. (2010). Adding instructional features that promote learning in a game-like environment. Journal of Educational Computing Research, 42(3), 241-265.

Moreno, R., \& Mayer, R. E. (2005). Role of guidance, reflection, and interactivity in an agentbased multimedia game. Journal of Educational Psychology, 97(1), 117-128. 
Niehaus, J., \& Riedl, M. O. (2009). Scenario adaptation: An approach to customizing computerbased training games and simulations. In Proceedings of the AIED 2009 Workshop on Intelligent Educational Games (Vol. 3, pp. 89-98). An approach to customizing computer-based training games and simulations. In Proceedings of the AIED 2009 Workshop on Intelligent Educational Games (Vol. 3, pp. 89-98).

O’Neil, H.F., Chung, G.K.W.K., Kerr, D., Vendlinksi, T.P., Buschang, R.E., \& Mayer,R.E. (2014). Adding self-explanation prompts to an educational computer game. Computers in Human Behavior, 30, 23-28.

Parnafes, O., \& Disessa, A. (2004). Relations between types of reasoning and computational representations. International Journal of Computers for Mathematical Learning, 9(3), 251-280.

Reif, F., \& Scott, L. A. (1999). Teaching scientific thinking skills: Students and computers coaching each other. American Journal of Physics, 67(9), 819-831.

Ringenberg, M. A., \& VanLehn, K. (2006, June). Scaffolding problem solving with annotated, worked-out examples to promote deep learning. In International Conference on Intelligent Tutoring Systems (pp. 625-634). Springer Berlin Heidelberg.

Rowe, J. P., \& Lester, J. C. (2015, June). Improving student problem solving in narrativecentered learning environments: A modular reinforcement learning framework. In International Conference on Artificial Intelligence in Education (pp. 419-428). Springer International Publishing. 
Roy, M., \& Chi, M. T. H. (2005). The self-explanation principle in multimedia learning. In R. E. Mayer (Ed.), The Cambridge handbook of multimedia learning (pp. 271-286). New York: Cambridge University Press.

Sampayo-Vargas, S., Cope, C. J., He, Z., \& Byrne, G. J. (2013). The effectiveness of adaptive difficulty adjustments on students' motivation and learning in an educational computer game. Computers \& Education, 69, 452-462.

Soflano, M., Connolly, T. M., \& Hainey, T. (2015). Learning style analysis in adaptive GBL application to teach SQL. Computers \& Education, 86, 105-119.

van Oostendorp, H., van der Spek, E.D. \& Linssen, J. (2014).Adapting the Complexity Level of a Serious Game to the Proficiency of Players. European Alliance for Innovation Endorsed Transactions on Serious Games, 1 (2), (pp. 1-8).

VanLehn, K. (2011). The relative effectiveness of human tutoring, intelligent tutoring systems, and other tutoring systems. Educational Psychologist, 46(4), 197-221.

Wouters, P., van Nimwegen, C., van Oostendorp, H., \& van der Spek, E. D. (2013). A metaanalysis of the cognitive and motivational effects of serious games. Journal of Educational Psychology, 105, 249-265. doi:10.1037/a0031311

Young, M. F., Slota, S., Cutter, A. B., Jalette, G., Mullin, G., Lai, B., .. Yukhymenko, M. (2012). Our princess is in another castle: A review of trends in serious gaming for education. Review of Educational Research, 82, 61-89. doi:10.3102/0034654312436980 


\section{Tables and Figures}

Table 1. ANOVAs Comparing Overall Performance Metrics by Condition

\begin{tabular}{|c|c|c|c|c|c|c|c|c|c|}
\hline & \multicolumn{2}{|c|}{ Nav-Only } & \multicolumn{2}{|c|}{ Nav+Abstract } & \multicolumn{2}{|c|}{$\mathrm{Nav}+$ Adaptive } & \multirow[b]{2}{*}{$\mathrm{F}$} & \multirow[b]{2}{*}{$\mathrm{p}$} & \multirow{2}{*}{$\begin{array}{c}\text { Significant } \\
\text { Tukey HSDs } \\
\text { \& Effect Sizes }\end{array}$} \\
\hline $\begin{array}{c}\text { Performance } \\
\text { Metric }\end{array}$ & $\mathrm{M}$ & SD & M & SD & M & SD & & & \\
\hline Pre-Test score & 3.864 & 2.216 & 3.925 & 2.623 & 4.974 & 3.132 & $\begin{array}{c}\mathrm{F}(2,132)= \\
2.248\end{array}$ & .110 & --- \\
\hline $\begin{array}{c}\text { Post-Test } \\
\text { score }\end{array}$ & 8.182 & 3.768 & 6.981 & 3.815 & 8.974 & 3.745 & $\begin{array}{c}\mathrm{F}(2,132)= \\
3.215\end{array}$ & .043 & $\begin{array}{l}\text { NAdap }>\text { NAbstr } \\
\text { Cohen } d=0.53\end{array}$ \\
\hline $\begin{array}{l}\text { Highest } \\
\text { Level } \\
\text { Completed }\end{array}$ & 19.77 & 4.377 & 15.60 & 4.753 & 18.08 & 4.181 & $\begin{array}{c}F(2,132)= \\
10.658\end{array}$ & .000 & $\begin{array}{l}\text { NOnly }>\text { NAbstr } \\
\text { Cohen } d=0.91 \\
\text { NAdap }>\text { NAbstr } \\
\text { Cohen } d=0.55\end{array}$ \\
\hline $\begin{array}{c}\text { Total } \\
\text { Engagement }\end{array}$ & 9.568 & 3.323 & 10.038 & 3.281 & 9.421 & 3.072 & $\begin{array}{c}\mathrm{F}(2,132)= \\
.465\end{array}$ & .629 & --- \\
\hline $\begin{array}{c}\text { Average } \\
\text { Actions Used } \\
\text { per Attempt }\end{array}$ & 5.301 & 2.422 & 9.938 & 2.902 & 9.203 & 2.34 & $\begin{array}{l}F(2,132) \\
=42.009\end{array}$ & $\begin{array}{c}<.00 \\
1\end{array}$ & $\begin{array}{l}\text { NAdap }>\text { NOnly } \\
\text { Cohen } d=1.64 \\
\text { NAbstr }>\text { NOnly } \\
\text { Cohen } d=1.74\end{array}$ \\
\hline $\begin{array}{c}\text { Average } \\
\text { Attempts per } \\
\text { Level }\end{array}$ & 4.771 & 4.571 & 4.963 & 3.975 & 3.706 & 2.417 & $\begin{array}{l}\mathrm{F}(2,132) \\
=1.305\end{array}$ & .275 & --- \\
\hline
\end{tabular}

Table 2. Pre-Test Post-Test Comparisons Within Each Condition

\begin{tabular}{lllll}
\hline Condition & Pretest (SD) & Post Test (SD) & $\mathrm{p}$ & Effect size (Cohen's d) \\
\hline \hline Nav-Only & $3.864(2.216)$ & $8.182(3.768)$ & $<0.001$ & Post $>$ Pre: 1.309 \\
Nav+Adaptive & $4.974(3.132)$ & $8.974(3.745)$ & $<0.001$ & Post $>$ Pre: 1.151 \\
Nav+Abstract & $3.925(2.623)$ & $6.981(3.815)$ & $<0.001$ & Post $>$ Pre: 0.904 \\
\hline
\end{tabular}


Table 3. Pearson Correlations between Engagement and Post-Test Score

\begin{tabular}{|l|l|l|l|l|l|l|}
\hline \multirow{2}{*}{ Condition } & Engagement & Post-test & $\begin{array}{l}\text { Pearson } \\
\text { Correlation }\end{array}$ & $\mathrm{p}$ \\
\cline { 2 - 7 } & $\mathrm{M}$ & $\mathrm{SD}$ & $\mathrm{M}$ & $\mathrm{SD}$ & -0.142 & .357 \\
\hline Nav-Only & 9.568 & 3.323 & 8.182 & 3.768 & -0.233 & .093 \\
\hline Nav+Abstract & 10.038 & 3.281 & 6.981 & 3.815 & -0.196 & .237 \\
\hline Nav+Adaptive & 9.421 & 3.072 & 8.974 & 3.745 & \multicolumn{2}{l}{} \\
\hline
\end{tabular}

Table 4. Pearson Correlations between Engagement and Highest Level Completed

\begin{tabular}{|c|c|c|c|c|c|c|}
\hline \multirow{2}{*}{ Condition } & \multicolumn{2}{|c|}{ Engagement } & \multicolumn{2}{|c|}{ Highest level Completed } & \multirow{2}{*}{$\begin{array}{l}\text { Pearson } \\
\text { Correlation }\end{array}$} & \multirow[b]{2}{*}{$\mathrm{p}$} \\
\hline & M & SD & $\mathrm{M}$ & SD & & \\
\hline Nav-Only & 9.568 & 3.323 & 19.77 & 4.377 & -0.276 & 0.070 \\
\hline $\mathrm{Nav}+$ Abstract & 10.038 & 3.281 & 15.60 & 4.753 & -0.402 & 0.003 \\
\hline $\mathrm{Nav}+$ Adaptive & 9.421 & 3.072 & 18.08 & 4.181 & -0.693 & $<0.001$ \\
\hline
\end{tabular}

Table 5. Repeated Measures ANOVA of The Low, Medium, and High-abstraction Explanation Prompts Within the Nav-Adaptive Condition

\begin{tabular}{|c|c|c|c|c|c|c|c|c|c|}
\hline & \multicolumn{2}{|c|}{$\begin{array}{l}\text { Low- } \\
\text { Abstraction }\end{array}$} & \multicolumn{2}{|c|}{$\begin{array}{l}\text { Med- } \\
\text { Abstraction }\end{array}$} & \multicolumn{2}{|c|}{$\begin{array}{l}\text { High- } \\
\text { Abstraction }\end{array}$} & \multirow[t]{2}{*}{$\mathrm{F}$} & \multirow[t]{2}{*}{$\mathrm{p}$} & \multirow{2}{*}{$\begin{array}{l}\text { Multiple Comparisons } \\
\text { (Bonferroni corrected) }\end{array}$} \\
\hline & $\mathrm{M}$ & SD & $\mathrm{M}$ & SD & $\mathrm{M}$ & SD & & & \\
\hline $\begin{array}{l}\text { Nav+Adap } \\
\text { tive }\end{array}$ & 107.4 & 6.42 & 103.9 & 6.30 & 113.2 & 6.69 & $\begin{array}{l}F(2,115) \\
=10.153\end{array}$ & 0.002 & $\begin{array}{l}\text { High }>\text { Low } \\
\text { p }<0.001 \\
\text { High }>\text { Medium } \\
p=0.001\end{array}$ \\
\hline
\end{tabular}




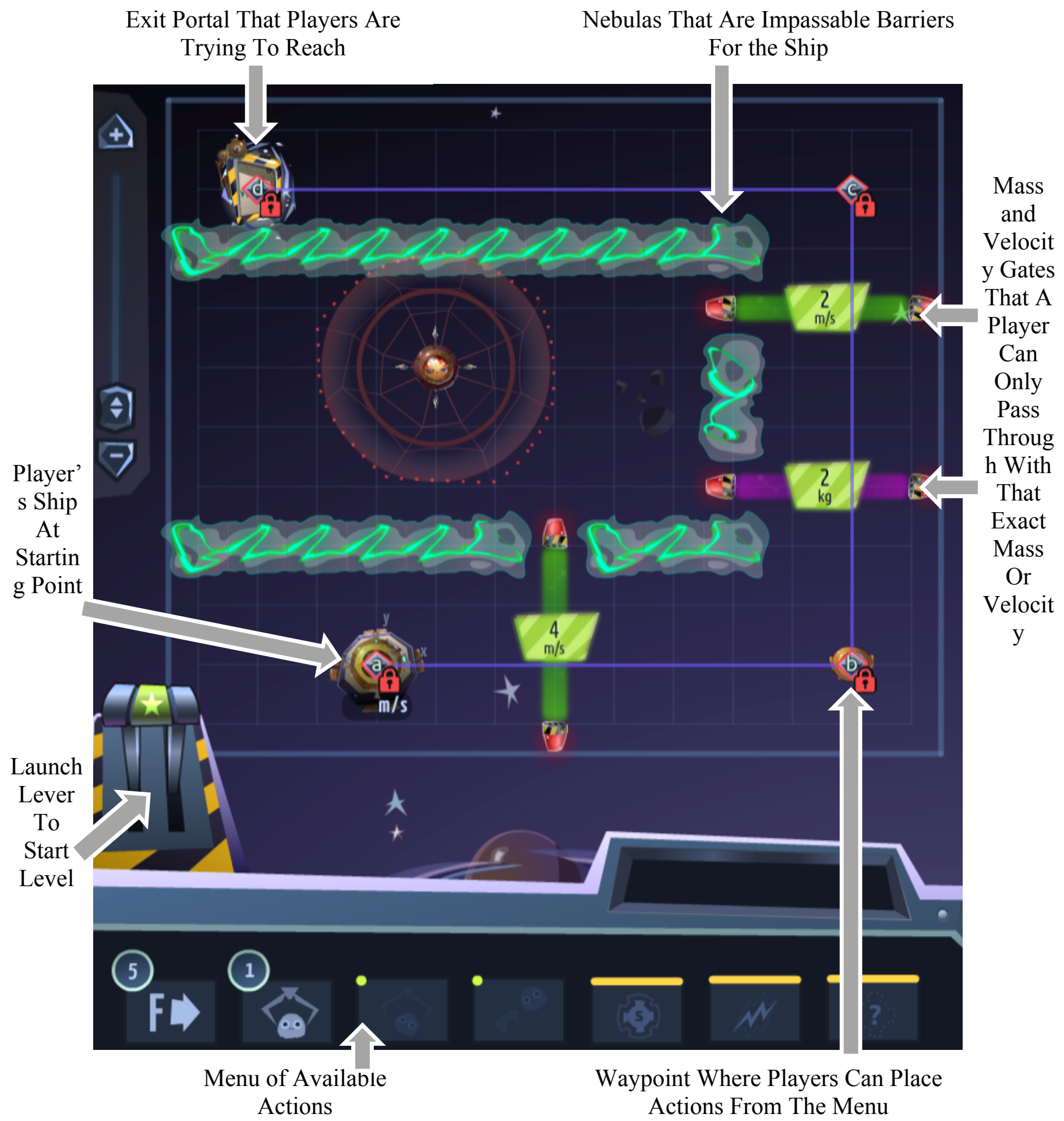

Figure 1. Anatomy of an EPIGAME level. Players place and modify actions from the menu at various waypoints to create a travel plan to reach the Exit Portal. When the plan is ready, the player pulls the launch lever. Actions are activated when and if the ship reaches the waypoint where they have been placed. 


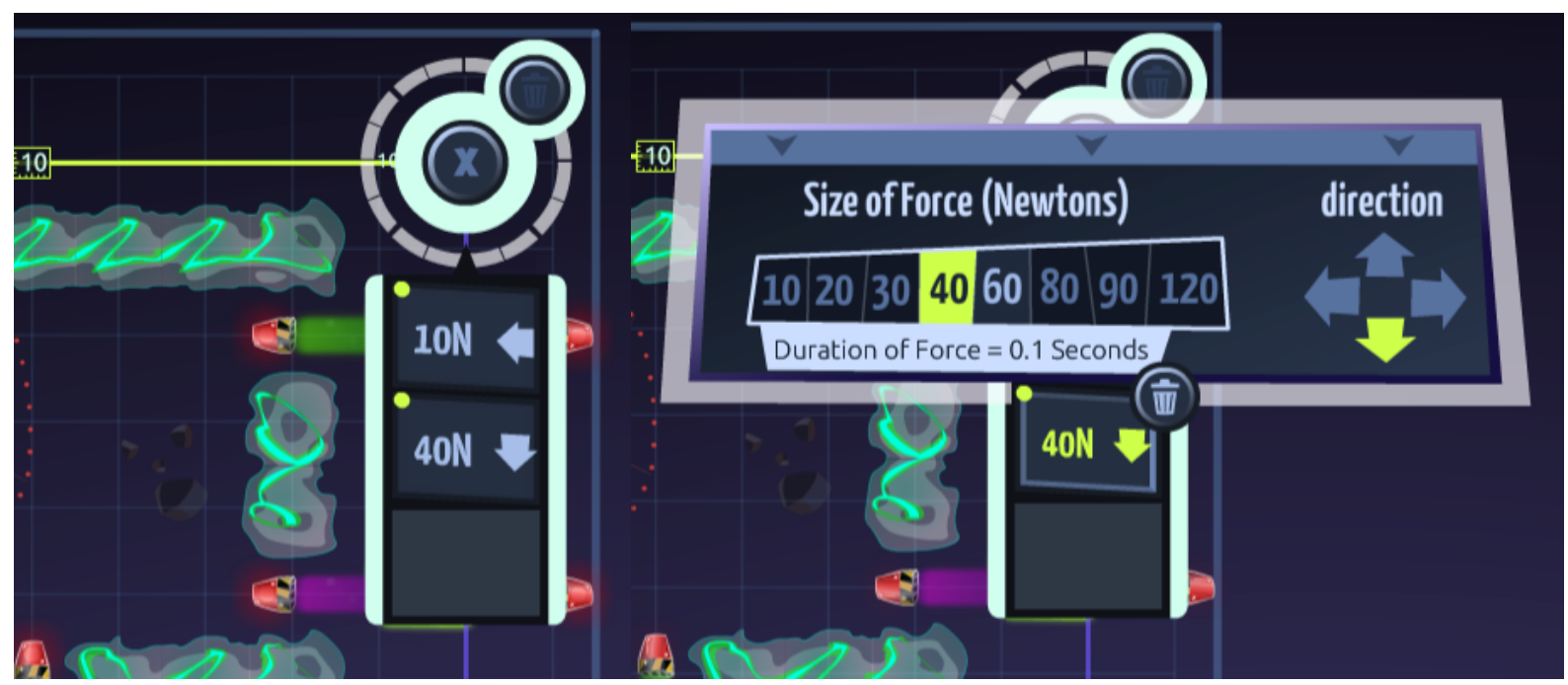

Figure 2. Clicking on a waypoint on the map shows the commands currently at that waypoint (left). Force commands can be added and edited in terms of magnitude and direction (right). Other commands can be added in terms of picking up, dropping, or throwing masses (fuzzies), which, in turn, affect the mass and/or velocity of the player's ship. 

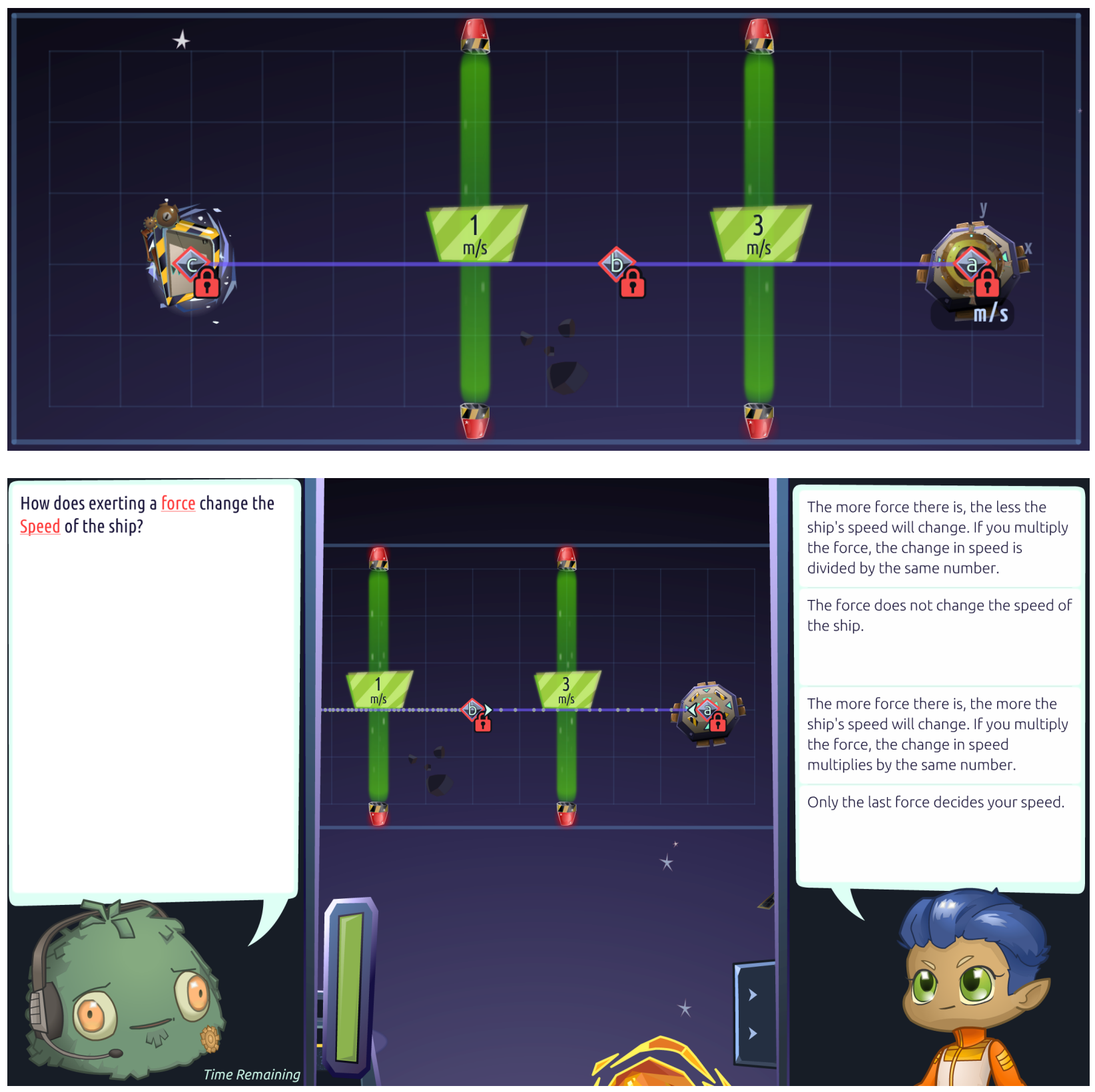

Figure 3. After the introductory dialog in a warp mission, students encounter the navigation phase of the warp mission (top). After succeeding in the navigation phase in a warp mission, students encounter the explanation phase of the warp mission (bottom). 
Question 14

A $1 \mathrm{~kg}$ alien is holding onto a $1 \mathrm{~kg}$ asteroid in space. They are moving to the left at $2 \mathrm{~m} / \mathrm{s}$.

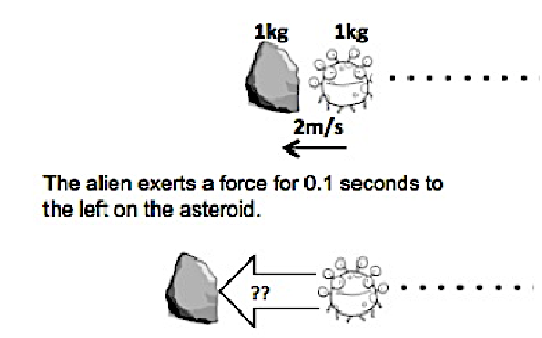

The alien is now stopped.
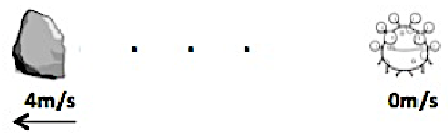

How much force was used by the alien to push the asteroid?
Please circle the best possible answer from the options below

A. $20 \mathrm{~N}$

B. $4 \mathrm{~N}$

C. $\quad 2 \mathrm{~N}$

D. $40 \mathrm{~N}$

Figure 4. Example Assessment Question 